\title{
On the radio emitting high mass X-ray binary LS 5039
}

\author{
J. S. Clark ${ }^{1,2}$, P. Reig ${ }^{3,4}$, S. P. Goodwin ${ }^{1,5}$, V. M. Larionov ${ }^{6,7}$, P. Blay $^{8}$, M. J. Coe ${ }^{9}$, J. Fabregat ${ }^{8}$, \\ I. Negueruela ${ }^{10}$, I. Papadakis ${ }^{3}$, and I. A. Steele ${ }^{11}$ \\ 1 Astronomy Centre, CPES, University of Sussex, Brighton, BN1 9QH, UK \\ 2 Department of Physics and Astronomy, University College London, Gower Street, London, WC1E 6BT, \\ England, UK \\ 3 Physics Department, University of Crete, PO Box 2208, 71003, Heraklion, Greece \\ 4 Foundation for Research and Technology-Hellas, 71110, Heraklion, Greece \\ 5 Department of Physics and Astronomy, University of Wales, Cardiff, CF24 3YB, Wales, UK \\ ${ }^{6}$ Astronomical Institute of St. Petersburg University, St. Petersburg 198904, Russia \\ 7 Isaac Newton Institute of Chile, St. Petersburg Branch \\ 8 Departmento de Astronomia, Universidad de Valencia, 46100 Burjassot, Valencia, Spain \\ 9 Department of Physics \& Astronomy, University of Southampton, Highfield, Southampton, SO17 1BJ, UK \\ 10 Observatoire de Strasbourg, 11 rue de l'Université, 67000 Strasbourg, France \\ 11 Astrophysics Research Institute, Liverpool John Moores University, Liverpool, L41 1LD, UK
}

Received 24 April 2001 / Accepted 26 June 2001

\begin{abstract}
We present new optical - near-IR spectroscopic and photometric observations of the newly discovered galactic microquasar LS 5039, which indicate a classification for the mass donor in the system of $\mathrm{O} 6.5 \mathrm{~V}((\mathrm{f}))$. Optical spectroscopy and photometry shows no variability over a timescale of years, and we find no evidence of modulation by, or emission from the compact companion in these data. However significant photometric variability ( 0.4 mag) is present in the $H$ and $K$ bands between 1995-2000. Such variability has been observed in other radio bright X-ray binaries where it has been attributed to synchrotron emission from the jet. However, given the non-thermal spectral index of the radio emission in LS 5039 this explanation appears unlikely, predicting a near-IR flux $\sim 3$ orders of magnitude too small to contribute significantly at such wavelengths. Nightly optical photometry over a 21 day period between 2000 May-June reveals variability at a level of a few hundredths of a magnitude, with no periodicity or long term trend visible. Likewise, while the radio lightcurves show moderate variability $(\sim 20$ per cent of the mean flux density) we find no evidence of periodic modulation - Monte Carlo simulations constrain any such periodic variability to $<4$ per cent modulation at $2.25 \mathrm{GHz}$. The differences in behaviour between LS 5039 and Cygnus X-1 - the most closely related radio emitting High Mass X-ray Binary are likely to be a result of the weaker stellar wind and probable greater orbital separation of LS 5039 compared to Cyg X-1.
\end{abstract}

Key words. stars: early-type - stars: individual: LS 5039 - X-rays: stars, binaries

\section{Introduction}

The early type star LS 5039 has been proposed as the optical counterpart to the X-ray source RX J1826-1450 by Motch et al. (1997, hereafter M97), who classify it as $\mathrm{O} 7 \mathrm{~V}((\mathrm{f}))$ on the basis of low resolution blue end spectroscopy. Subsequent observations by Martí et al. (1998, hereafter Ma98) suggested that LS 5039/RX J1826-1450 was also associated with a persistent, moderately variable unresolved radio source with a non thermal radio spectrum of spectral index $\alpha \sim-0.47$ (adopting the convention $\left.S_{\nu} \propto \nu^{\alpha}\right)$.

Send offprint requests to: J. S. Clark, e-mail: jsc@star.ucl.ac.uk
Ribó et al. (1999, henceforth R99) present a detailed timing and spectral analysis of X-ray data obtained with the Rossi X-ray Timing Explorer (RXTE). They find the timing analysis shows no evidence for either pulsed emission, or periodic variability between 2-200 d, which might arise from orbital modulation of the X-ray flux. Furthermore, they analysed four months of daily observations of the 8.3 and $2.25 \mathrm{GHz}$ flux of LS 5039, obtained as part of a long term monitoring campaign with the Green Bank Interferometer (GBI) ${ }^{1}$, but found no evidence for periodic variability between $2-50 \mathrm{~d}$, or well defined flaring activity in these data.

\footnotetext{
1 The Green Bank Interferometer is a facility of the USA National Science Foundation operated by NRAO in support of the NASA High Energy Astrophysics programs.
} 
More recently Paredes et al. (2000) succesfully resolved the radio emission as a bipolar jet, confirming LS5039 as one of the few radio emitting High Mass X-ray Binaries (hereafter HMXB) known in the galaxy. Given their rather heterogeneous nature, further observations of RX J1826-1450/LS 5039 are clearly of interest. Here we present new optical (4100-7400 $\AA$ ) and near-IR (1.5$2.2 \mu \mathrm{m})$ spectroscopic data obtained between 1995-2000 enabling a more accurate spectral classification for the system. Photometric observations from 1997-2000 are also presented. In combination with published data and our spectroscopic observations these allow us to search for medium-long term variability. Finally we also undertake a more detailed analysis of the complete 8.3 and $2.25 \mathrm{GHz}$ GBI datasets than has previously been published.

\section{Spectroscopic observations}

Optical spectroscopic observations were made with the $1.9 \mathrm{~m}$ telescope at the South African Astronomical Observatory (SAAO) on 1997 June 23, the $1.3 \mathrm{~m}$ telescope at Mount Skinakas (Crete, Greece) on 1999 July 27 and 2000 July 20-22 and the ESO 1.52-m telescope at La Silla Observatory, Chile on 2000 September 13. The spectrum at SAAO was taken with the ITS spectrograph + SITe CCD + 1200 lines $\mathrm{mm}^{-1}$ (grating No 4) $+250 \mu \mathrm{m}$ slit. With this set-up the dispersion was $\sim 0.5 \AA$ /pixel and the wavelength coverage 4100-4900 $\AA$. The f/7.7 RitcheyChretien telescope at Skinakas observatory was equipped with a $2000 \times 800$ ISA SITe CCD + 1302 lines $\mathrm{mm}^{-1}$ grating, giving a dispersion of $\sim 1 \AA /$ pixel. In the 1999 July spectrum the slit width was $320 \mu \mathrm{m}$, whereas in the 2000 July spectra it was $80 \mu \mathrm{m}$. Finally the $1.52 \mathrm{~m} \mathrm{La}$ Silla Observatory telescope was equipped with the Boller and Chivens spectrograph $+\# 33$ holographic grating and the Loral 38 camera. This configuration gives a dispersion of $\sim 1.0 \AA /$ pixel (resolution of $\approx 3 \AA$ at $\sim 4500 \AA$ ).

Near-IR observations of LS 5039 were made with the United Kingdom Infrared Telescope (UKIRT) on 1999 July 22-24, using the Cooled Grating Spectrometer (CGS4). Observations between 1.5-2.2 microns were made using the long focal length camera plus the 150 line $/ \mathrm{mm}$ grating, giving a velocity resolution of $\sim 50 \mathrm{~km} \mathrm{~s}^{-1}$. Note that at this resolution the wavelength range of a single spectrum is $\sim 0.08 \mu \mathrm{m}$ - hence breaks in the spectra presented in Fig. 3 are due to slight missmatches in wavelength coverage between individual spectra. Initial data reduction was carried out at the telescope using the CGS4DR software (Puxley et al. 1992). This removes bad pixels, debiases, flat-fields, linearity corrects and interleaves oversampled scan positions. The subsequent stages of data reduction, comprising of sky subtraction, extraction, derippling and wavelength calibration using observations of a CuAr lamp, were carried out using the Starlinksupported package FIGARO. Removal of telluric features was accomplished via the procedure described by Clark \& Steele (2000).
Table 1. Equivalent widths of the identified lines shown in Figs. 1 and 2. Errors are $<10 \%$.

\begin{tabular}{lccc}
\hline Line & \multicolumn{3}{c}{$E W(\AA)$} \\
& 1997 & 1999 & 2000 \\
\hline H $\delta$ & 2.3 & & 2.2 \\
HeII $\lambda 4200$ & 0.50 & & 0.55 \\
H $\gamma$ & 2.4 & & 2.4 \\
HeI $\lambda 4471$ & 0.50 & & 0.50 \\
HeII $\lambda 4541$ & 0.92 & & 0.90 \\
HeII $\lambda 4686$ & 0.95 & & 1.0 \\
H $\beta$ & & 2.4 & 2.5 \\
HeI $\lambda 6406$ & & 0.24 & 0.26 \\
H $\alpha$ & & 3.6 & 3.9 \\
HeI $\lambda 6678$ & & 0.90 & 0.85 \\
HeI $\lambda 7065$ & & 0.50 & 0.55 \\
\hline
\end{tabular}

\subsection{Spectral classification}

The optical Balmer lines are in absorption, indicating the absence of a dense circumstellar envelope around the star, and precluding an Oe/Be classification. The red-end spectrum is characterised by the presence of numerous interstellar lines (we derive reddening estimates from these in Sect. 4) and neutral helium lines in absorption ( $\lambda$ 5876, 6406, 6679 and 7065). The blue-end of the optical spectrum is dominated by the hydrogen Balmer series and by strong He II lines. The ratio between He II 4541 and He I 4471 corresponds to a spectral type O6.5, according to the relations of Mathys (1988). The fact that He II 4686 is strongly in absorption identifies the star as main-sequence. This can be checked by direct comparison with MK standards. As seen in Fig. 1, the strength of the He I and He II lines in LS 5039 falls between those of the $\mathrm{O} 6 \mathrm{~V}((\mathrm{f}))$ and O7V((f)) standards. Very weak N III $\lambda 4634-40-42$ emission is present. The combination of strong He II absorption and weak $\mathrm{N}$ III emission is represented by $((f))$ - suggesting an O6.5V((f)) classification - slightly earlier than the classification of M97, made with a single low resolution $\lambda 3800$ 4600 spectrum.

The spectral classification of hot, luminous stars via near-IR spectroscopy alone is far less precise than via conventional optical classification. Nevertheless, it provides an independent test of the optical classification. The comprehensive spectral atlas of Hanson et al. (1997) was used for classification of the $K$ band spectrum of LS 5039 . The strength of H I $2.166 \mu \mathrm{m}$ and $\mathrm{He}$ II $2.1885 \mu \mathrm{m}$ lines (Table 2) restrict the spectral type to later than O6, and earlier than O8, while the He I $2.113 \mu \mathrm{m}$ absorption feature further constrains the spectral type to earlier than O7.5. Finally, the N III emission line is absent in normal Main Sequence O6-O8 spectra and confirms the $\mathrm{O}((\mathrm{f}))$ classification, fully consistent with the optical classification.

Unfortunately, no such comprehensive reference work exists for the classification of OB stars via the $H$ band; 


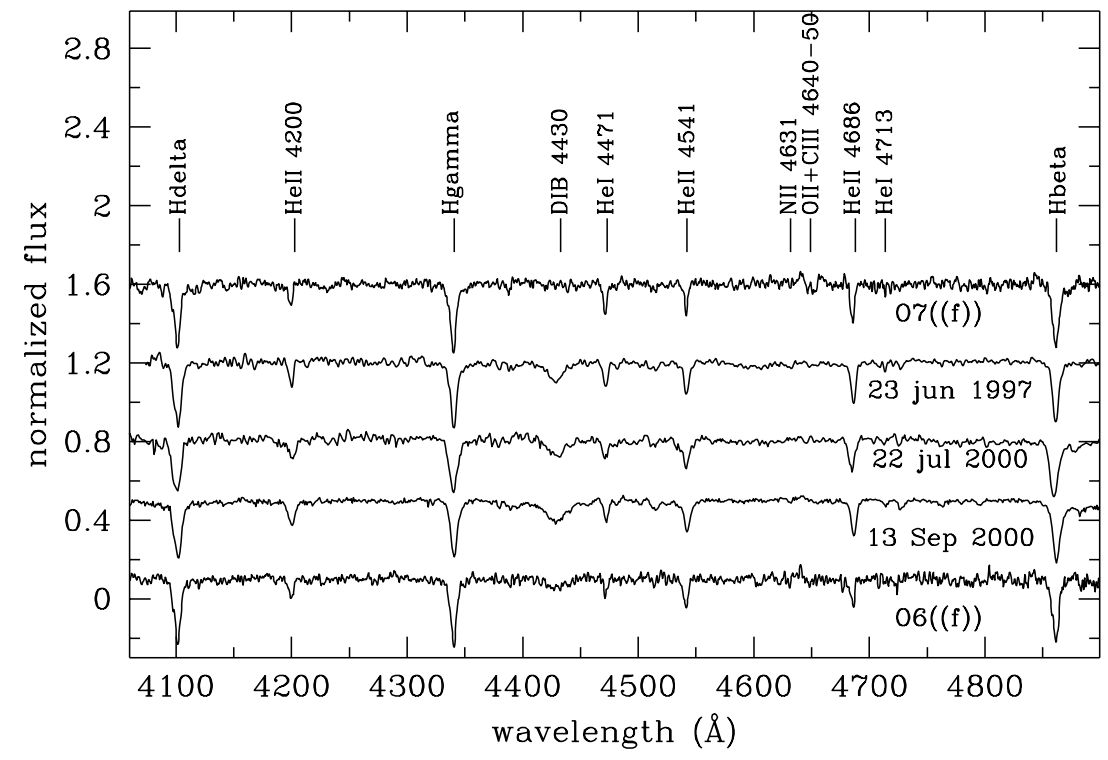

Fig. 1. Blue end (4050-4950 $\AA$ ) spectra of LS 5039 with line identifications indicated (Diffuse Interstellar bands = DIB). The comparison stars are the $\mathrm{O} 6 \mathrm{~V}((\mathrm{f}))$ and $\mathrm{O} 7 \mathrm{~V}((\mathrm{f}))$ standards from the digital atlas of Walborn \& Fitzpatrick (1990; HD 101190 and 15 Mon respectively).

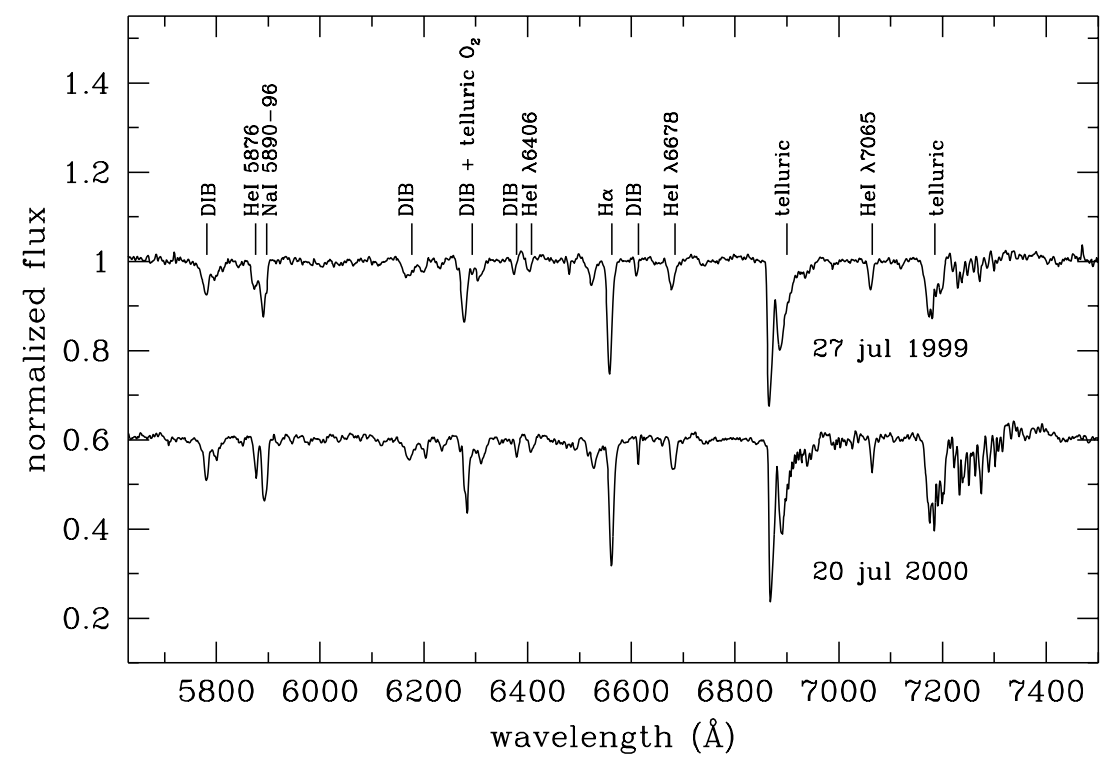

Fig. 2. Red end (5650-7500 $\AA)$ spectrum of LS 5039.

we use the preliminary work of Blum et al. (1997) and the more extensive work of Hanson et al. (1998), noting that this atlas is restricted to a narrow waveband (1.66$1.72 \mu \mathrm{m}$ ) and complete only for spectral types between O7-B9. Comparison of the strengths of the H I brackett series to the spectra presented by Blum et al. (1997) confirm an $\mathrm{O}$ star classification, while the strength of the He II transition is consistent with a spectral type of O8 or earlier (Hanson et al. 1998). Direct comparison to the spectrum of the similar O7V((f)) star HD 47839 presented in Hanson et al. (1998) shows the He II feature to be stronger for LS 5039, while the H I Brackett 11 line is somewhat weaker. However He I absorption is of comparable strength in both spectra.

In conclusion we find that both $H$ and $K$ band spectra are consistent with the optical classification of LS 5039 as $\mathrm{O} 6.5 \mathrm{~V}((\mathrm{f}))$, with the $K$ band spectrum providing the more rigorous constraints to spectral type (i.e. no earlier than O6.5 and no later than O7) We note that we find no evidence for a luminous stellar companion for LS 5039 in any of the spectra, nor any additional emission features that might be attributed to emission from a jet or accretion disc.

\section{Photometry}

\subsection{Long term variability}

Broadband photometric observations of LS 5039 from 1973-present have been reported by a number of authors (e.g. Drilling 1975; Lahulla \& Hilton 1992, Ma98) and indicate that between 1973-present LS 5039 has been remarkably stable (e.g. $\Delta V<0.1$ mag.) in the optical. This stability is also evident in the observations presented 

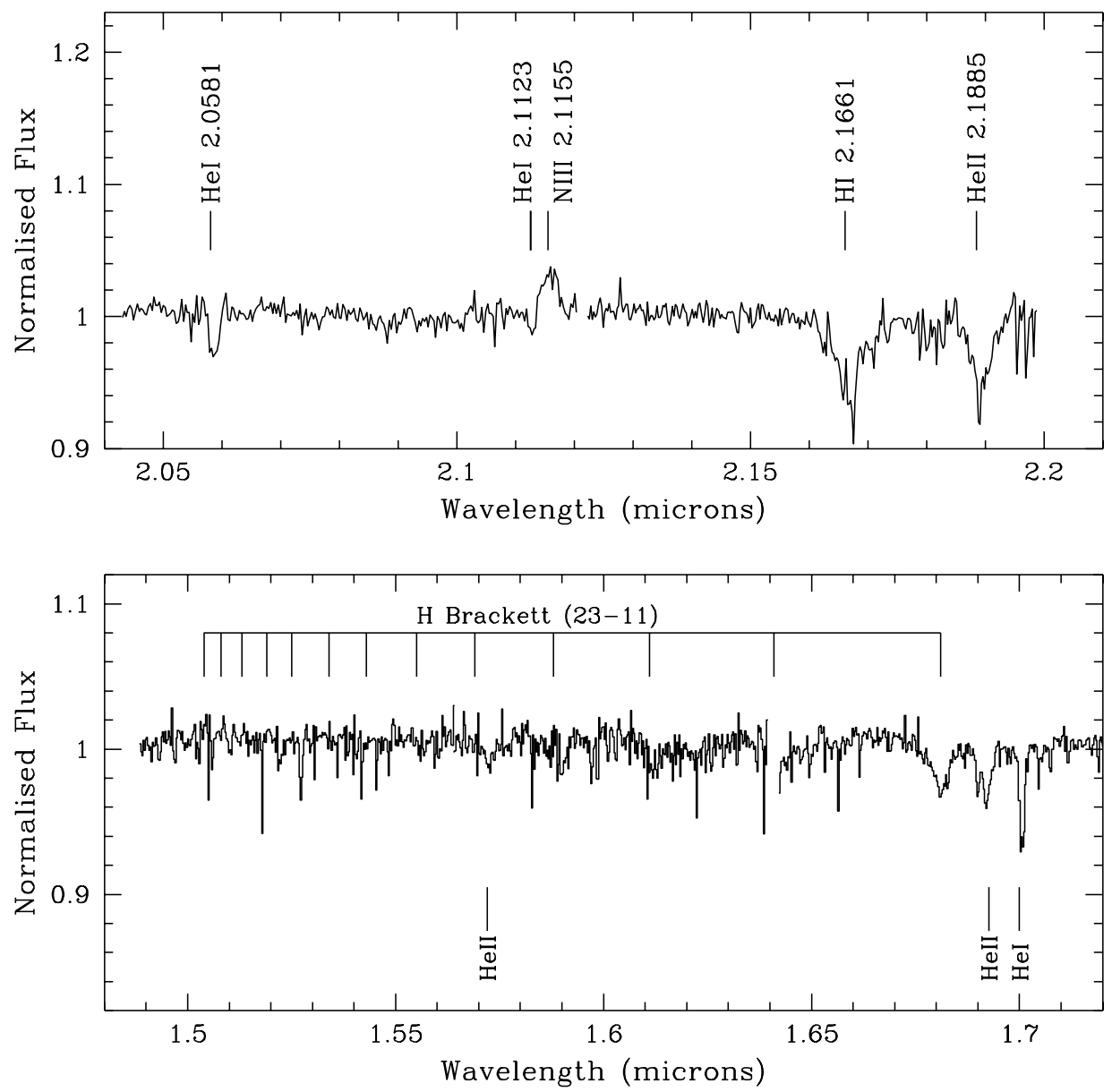

Fig. 3. Composite $K$ band $(2.05-2.20 \mu \mathrm{m})$ and $H$ band $(1.49-1.72 \mu \mathrm{m})$ spectra of LS 5039; wavelength given in $\mu \mathrm{m}$. Note the break between the 2 spectra at $2.125 \mu \mathrm{m}$ in the $K$ band and at $1.64 \mu \mathrm{m}$ in the $H$ band; unfortunately the latter break obscures

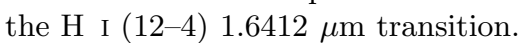

Table 2. Line identifications for LS 5039 in the range 1.49-1.72 and $2.05-2.20 \mu \mathrm{m}$. Note the $E W$ quoted for the line identified with He II $1.693 \mu \mathrm{m}$ also includes a contribution from the feature visible in the blue wing of the profile. Errors are estimated at $20 \%$ of the quoted $E W$.

\begin{tabular}{ccc}
\hline $\begin{array}{c}\text { Wavelength } \\
\text { (microns) }\end{array}$ & $\begin{array}{c}\text { Feature } \\
\text { (microns) }\end{array}$ & $\begin{array}{c}E W \\
(\AA)\end{array}$ \\
\hline 1.573 & He II $\lambda 1.572$ & 0.5 \\
1.612 & H I $\lambda 1.611$ & 0.8 \\
1.681 & H I $\lambda 1.681$ & 1.6 \\
1.690 & $?$ & Blend \\
1.692 & He II $\lambda 1.693$ & 1.4 \\
1.701 & He I $\lambda 1.701$ & 1.1 \\
2.059 & He I $\lambda 2.058$ & 0.5 \\
2.113 & He I $\lambda 2.113$ & 0.1 \\
2.116 & N III $\lambda 2.116$ & -1.1 \\
2.167 & H I $\lambda 2.166$ & 2.6 \\
2.189 & He II $\lambda 2.189$ & 2.0 \\
\hline
\end{tabular}

in Table 3 - obtained by us on 1996 October 3-5 with the $1.9 \mathrm{~m}$ telescope of the South African Astronomical
Observatory (SAAO) and 2000 August 17-24 with the $0.7 \mathrm{~m}$ telescope of the Crimean Astrophysical Observatory (CrAO). With the exception of the single $V$ band datum from Kilkenny et al. 1993 these observations are consistent with no variability.

However JHK band observations made with the Telescope Carlos Sanchez (TCS) between 1995-2000 (Table 4) appear to show significant variability ( $0.4 \mathrm{mag})$ in $H$ and $K$ bands respectively. The same standard star (BS 7236) and reduction procedures were adopted for all observations, and given the proximity of BS 7236 to LS 5039 the possibility of differential extinction due to dust close to the horizon can also be eliminated as a cause of such variability. The source of such variability is not clear. The wind from the $\mathrm{O} 6.5 \mathrm{~V}((\mathrm{f}))$ primary is not expected to contribute significantly at these wavelengths (Runacres, priv. comm.), while extrapolation of the radio flux to near-IR wavelengths yields a flux 3 orders of magnitude too small to explain the observed variability.

\subsection{Medium term variability}

In order to search for medium term, day-to-day variability - possibly indicative of orbital modulation 
Table 3. Comparison of our 2 epochs of broad band photometry to archival observations presented by Kilkenny et al. (1993, K93), Spencer Jones et al. (1993, SJ93), both made with the SAAO \& Ma98 (1998 data).

\begin{tabular}{lcccccccc}
\hline Observation & $B$ & $V$ & $R$ & $I$ & $J$ & $H$ & $K$ & $L$ \\
\hline K93, SJ93 & - & $11.24 \pm 0.01$ & $10.59 \pm 0.01$ & $9.88 \pm 0.01$ & $9.02 \pm 0.01$ & $8.79 \pm 0.02$ & $8.57 \pm 0.03$ & - \\
1996 Oct. & $12.18 \pm 0.02$ & $11.33 \pm 0.02$ & $10.65 \pm 0.02$ & $9.87 \pm 0.02$ & $9.05 \pm 0.02$ & $8.75 \pm 0.02$ & $8.60 \pm 0.02$ & $8.69 \pm 0.05$ \\
1998 June 7 & - & $11.35 \pm 0.03$ & $10.64 \pm 0.03$ & $9.90 \pm 0.03$ & - & - & - & - \\
1998 June 8 & - & $11.39 \pm 0.03$ & $10.69 \pm 0.03$ & $9.95 \pm 0.03$ & - & - & - & - \\
2000 Sept. & $12.17 \pm 0.03$ & $11.32 \pm 0.01$ & $10.61 \pm 0.01$ & $9.91 \pm 0.01$ & - & - & - \\
\hline
\end{tabular}

Table 4. Broad band JHK photometry obtained with the TCS in the period 1995-2000.

\begin{tabular}{lccc}
\hline Date & $J$ & $H$ & $K$ \\
\hline $14 / 10 / 95$ & $9.06 \pm 0.01$ & $9.04 \pm 0.01$ & $8.93 \pm 0.03$ \\
$31 / 7 / 96$ & $9.05 \pm 0.03$ & $8.93 \pm 0.03$ & $8.85 \pm 0.03$ \\
$19 / 7 / 97$ & $9.06 \pm 0.01$ & $8.91 \pm 0.01$ & $8.88 \pm 0.01$ \\
$21 / 7 / 97$ & $9.07 \pm 0.02$ & $8.90 \pm 0.02$ & $8.83 \pm 0.02$ \\
$26 / 7 / 99$ & $9.04 \pm 0.02$ & $8.91 \pm 0.02$ & $8.82 \pm 0.01$ \\
$28 / 7 / 99$ & $9.10 \pm 0.02$ & $9.04 \pm 0.02$ & $9.01 \pm 0.02$ \\
$31 / 7 / 99$ & $8.97 \pm 0.02$ & $8.71 \pm 0.02$ & $8.58 \pm 0.02$ \\
$2 / 10 / 99$ & $9.01 \pm 0.02$ & $8.76 \pm 0.02$ & $8.63 \pm 0.02$ \\
$7 / 10 / 99$ & $9.02 \pm 0.02$ & $8.74 \pm 0.02$ & $8.55 \pm 0.02$ \\
$6 / 7 / 00$ & $8.99 \pm 0.02$ & $8.75 \pm 0.02$ & $8.65 \pm 0.04$ \\
$17 / 10 / 00$ & $9.05 \pm 0.02$ & $8.81 \pm 0.02$ & $8.65 \pm 0.01$ \\
$18 / 10 / 00$ & $8.97 \pm 0.02$ & $8.68 \pm 0.02$ & $8.53 \pm 0.01$ \\
\hline
\end{tabular}

- photometric observations of LS 5039 were made using the $1.3 \mathrm{~m}$ telescope at the Skinakas Observatory (Crete, Greece). Standard Johnson $B$ and $V$ filters were used in combination with a tektronix $1024 \times 1024$ CCD with $24 \mu \mathrm{m}$ pixels. The source was observed on 21 nights during the period May 12, 2000 to June 24, 2000. The seeing during the observation run varied between $\sim 1^{\prime \prime}$ and $\sim 3^{\prime \prime}$. Standard image processing (bias subtraction and flat-fielding) was applied to all images.

To improve the accuracy of the results we performed relative photometry of the source with respect to three reference stars in the CCD frame. We checked the constancy of these three stars by obtaining the difference between one of them and the average of the other two. The standard deviation of the individual light curves throughout the period was $\leq 0.01$. An idea of the accuracy of our relative photometry can be obtained by taking the standard deviation of the global light curve of the three reference stars: $\sigma_{B}=0.009$ and $\sigma_{V}=0.01$ for the $B$ and $V$ bands, respectively.

LS 5039 shows variations within a night with an amplitude of a few hundreths of magnitude. These low amplitude variations also occur from one night to the next but on a longer time scale no particular periodic or quasiperiodic trend is apparent. From these observations we can exclude the presence of (periodic) orbital modulation between 3-15 days, noting that if periodic variability were present at the level observed in HDE 226868 (Brocksopp et al. 2000) - the mass donor in Cygnus X-1 - our observations would have detected it. Aperiodic photometric variability at this level is not unknown in O type stars. Balona (1992) surveyed 16 bright O stars and found variability in all the supergiants observed, and a subset of the main sequence stars, including HD 101190, an O6V((f)) star (for which no periodicity was identifiable). Balona (1992) suggests that either variations in mass loss rates or non-radial pulsations could cause the observed variability but is unable to discriminate between the two possibilities.

\section{Interstellar reddening}

We have estimated the interstellar reddening to the source by using the correlation between the $E(B-V)$ colour excess and the equivalent width of diffuse absorption features of interstellar origin (Herbig 1975). We used the lines $\lambda 4430, \lambda 6376 / 79$ and $\lambda 6613$ only since a clean measurement of their equivalent width can be carried out. Due to the relative low resolution of our spectra some of the interstellar features appear blended with other interstellar or atmospheric lines. We find the mean colour excess to be $E(B-V)=0.9 \pm 0.2$, in agreement with an earlier measurement by M 97 of $E(B-V)=0.8 \pm 0.2$ using the same spectroscopic method. However we find that this estimate suggests a significant $(V-K) \sim 0.8$ colour excess when applied to the photometry from 1996 October (Table 3). By comparison we find that applying the photometrically derived reddening estimate from our data $(E(B-V)=$ $1.2 \pm 0.1)$ results in no excess emission in the near IR. Additionally, the X-ray data support a higher value for the colour excess. From a BeppoSAX observation a hydrogen column density of $\sim(1.0 \pm 0.3) \times 10^{22} \mathrm{~cm}^{-2}$ is found (Reig, private communication), implying $E(B-V)=1.9 \pm 0.6$ (Predehl \& Schmitt 1995) - not statistically different from the value adopted from the photometric data. We therefore choose to adopt $E(B-V)=1.2 \pm 0.1$ for LS 5039 given that it is consistent with both the near-IR colours of, and hydrogen column density to, LS 5039.

\section{Radio emission}

The results of GBI monitoring of LS 5039 have been presented by both Paredes et al. (2000) and R99. R99 analysed $\approx 130$ days worth of data but, while claiming moderate variability, could find no evidence of periodicity. 
Here we reanalyse the complete dataset (284 data points spanning 340 days between 1998 September 16-1999 August 22) taking into account the detection threshold at both wavelengths.

The $2.25 \mathrm{GHz}$ data is simple to analyse as it lies far above the significant detection limit of $10 \mathrm{mJy}$. The $2.25 \mathrm{GHz}$ light curve has a mean value of $31.3 \mathrm{mJy}$ and a standard deviation of $6.2 \mathrm{mJy}$; given that the noise level for this frequency is $4 \mathrm{mJy}$, the source is detected at the $\sim 8 \sigma$ level on average. The instrumental noise corrected source variance is $22.1 \mathrm{mJy}^{2}$. Therefore, the $\mathrm{rms}$ source variability amplitude at the $2.25 \mathrm{GHz}$ band is $\sim 15 \%$. This result is significant at a $95 \%$ confidence level (i.e. it is very likely that there is variability above the expected noise level).

The situation is different in the $8.3 \mathrm{GHz}$ band. The instrumental noise level (i.e. $\sigma$ ) is $6 \mathrm{mJy}$ and several data points in the light curve lie at or below this limit. In order to estimate the mean and variance of the light curve we assume that the signal is drawn from a Gaussian distribution, truncated at $10 \mathrm{mJy}$ (which is $\sim 1.8 \sigma_{\text {noise }}$ ) and minimise the likelihood function. Our best mean and standard deviation estimates are 13.7 and 6.7 mJy respectively (significantly different from the results if the truncation at $10 \mathrm{mJy}$ is not considered). Hence, the instrumental noise corrected source standard deviation is formally $2.3 \mathrm{mJy}$ (and the rms source variability amplitude $\sim 17 \%$ ) although this result is not statistically significant. We conclude that the rms variability amplitude of the source is similar in the two radio bands, although the result about the $8.3 \mathrm{GHz}$ band should be treated with caution as the source at that band is detected at only the $\sim 2 \sigma$ level on average.

Examination of the power spectra of the two sets of data show that there are no significant peaks. To determine what periodic signals could be hidden within the noise a series of Monte Carlo simulations were construced of a fake $2.25 \mathrm{GHz}$ data set drawing noise randomly from a gaussian with $\sigma=4 \mathrm{mJy}$ and adding a periodic signal of amplitude $4.7 \mathrm{mJy}$ (or less). Variability would be clear and unambiguous on periods of 2 day (limited by the sampling interval) to 340 days (the total data set) for a signal of $>1.2 \mathrm{mJy}$ amplitude, that no such signal is seen is very strong evidence that none exists. The $8.3 \mathrm{GHz}$ data is impossible to analyse in any significant way as the periodic signal (if there is one) is totally dominated by noise.

For this reason it is impossible to determine any information about the spectral index of the source. Monte Carlo simulations were used to construct fake data sets where the spectral index was known and then noise was added in the hope that there would be a difference between these fake data sets that would give an indication of the behaviour of the real source. Unfortunately the addition of the noise swamps any underlying behaviour making them indistinguishable. The binning of the data in order to (hopefully) minimise the noise does not help. Higher signal-to-noise observations are clearly required to make further progress in understanding the radio behaviour of LS 5039.
Table 5. Composition of known radio emitting HMXB (adapted from R99).

\begin{tabular}{lr}
\hline Source & $\begin{array}{r}\text { Primary }+ \\
\text { Secondary }\end{array}$ \\
\hline Cygnus X-3 & Wolf Rayet $+?$ \\
Cygnus X-1 & O9.7Iab +black hole \\
LSI+61 303 & Be + neutron star \\
SS 433 & OBe? +? \\
CI Cam & sgB $[\mathrm{e}]+?$ \\
LS 5039 & O6.5V $((\mathrm{f}))+?$ \\
\hline
\end{tabular}

\section{Discussion}

Currently radio emission is observed from $\sim 20$ per cent of all X-ray binaries, where it is thought to arise via the synchrotron mechanism in collimated jets. The number of confirmed radio emitting HMXB is still small, with only 5 other members of this rather heterogeneous group known in addition to LS 5039 (see Table 5), therefore it is of interest to compare the properties of LS 5039 to other members of this grouping.

Of these systems CI Cam has been observed to undergo only one radio outburst and the detection of apparently bipolar jets at this time has subsequently been questioned. Photometric monitoring of the post-outburst behaviour of CI Cam revealed a subsequent episode of dust formation (Clark et al. 2000) - which has not been observed in the other radio emitting HMXB - which raises the question of whether the radio emission arises in a jet or a less collimated outflow, such as is seen for dusty novae. Both Cyg X-3 and SS 433 exhibit episodes of aperiodic flaring which are not apparent in the radio lightcurves of LS 5039, most likely due to instabilities or structure in the wind of the primaries leading to variable accretion rates. LSI+61 303 shows periodic radio flaring at its orbital period - once again this appears to be related to the interaction of the accretor with the highly aspherical wind of the Be star primary. Quasi-periodic X-ray outbursts are a common feature of $\mathrm{Be} / \mathrm{X}$-ray binaries and are attributed to the interaction of the compact object with the dense circumstellar disc of the Be star primary around periastron. These observations suggest the wind geometry of the mass donors in HMXB plays a significant role in their X-ray and radio properties.

Since both LS 5039 and HDE 226868 (O9.7 Iab) - the mass donor in Cyg X-1 - are expected to have powerful quasi-spherical winds we might expect both systems to share similar properties. However, while both systems are persistent radio sources, Pooley et al. (1999) find periodic modulations at 5.6 days (orbital period) and $142 \pm 7$ days in the radio emission of Cyg X-1, which, if present at the same amplitude would have been detectable in LS 5039 (Sect. 5). Additionally, periodic modulation at the orbital period is also observed in the X-rays and $U B V J H K$ broad band photometry of Cyg X-1 
(Brocksopp et al. 2000) - again absent in LS 5039. We suggest that the differences in properties of the 2 systems are a product of differences in the stellar winds of both primaries in conjunction with a possible larger orbital separation for LS 5039 (see below).

Although the properties of the stellar wind of LS 5039 have yet to be determined (our optical spectra are not of sufficient resolution for detailed modeling), the terminal velocity, $V_{\infty}$, and mass loss rate, $\dot{M}$, for the slightly later O7V star HD 47839 are presented by Lamers \& Leitherer (1993). While $V_{\infty}=2300 \pm 200 \mathrm{~km} \mathrm{~s}^{-1}$ is within a factor of 2 of that of HDE $226868\left(V_{\infty}=1580 \mathrm{~km} \mathrm{~s}^{-1}\right.$, Gies \& Bolton 1986), $\log \dot{M}_{\mathrm{O} 7 \mathrm{~V}}=-6.3_{-0.41}^{+0.21} M_{\odot} \mathrm{yr}^{-1}$ is potentially an order of magnitude lower than that of HDE $226868\left(\log \dot{M}=-5.7 M_{\odot} \mathrm{yr}^{-1}\right.$; Gies \& Bolton 1986). Brocksopp et al. (2000) attribute the orbital modulation of the radio flux of Cyg X-1 to variable absorption by the dense stellar wind of HDE 226868 due to orbital motion of the compact companion. Using Eq. (4) of Leitherer et al. (1995) we find that the radio photosphere of LS 5039 is a factor of $\sim 4$ smaller than that of HDE 226868 (due to the $T^{-1 / 2}\left(\dot{M} / V_{\infty}\right)^{2 / 3}$ dependance of the radio photosphere radius) implying that modulation due to wind opacity is likely to be less important in LS 5039, resulting in weaker orbital modulation in the radio lightcurve (we also note that the linear size of the radio jets is larger than the radio photosphere of LS 5039; Paredes et al. 2000).

Based on the rate of the energy loss of electrons to Inverse Compton scattering in the UV radiation field of the star Paredes et al. (2000) suggest an orbital radius of $R_{\text {orb }} \sim 1.2 \times 10^{13} \mathrm{~cm}$ for the compact object in LS 5039 . The canonical mass and radius of an $06.5 \mathrm{~V}((\mathrm{f}))$ star are rather uncertain - here we adopt $R_{*}=10.3 R_{\odot}$ and $M_{*}=22 M_{\odot}$ (Puls et al. 1996). Then assuming a neutron star companion and applying Keplers Third Law we find a tentative orbital period of $\sim 54$ days and $R_{\text {orb }} / R_{*} \sim 21$ for LS 5039 compared to only $\sim 2.4$ for Cyg X-1. With such a small orbital separation in Cyg X-1 it is expected that both the stellar wind and star itself will be distorted by the presence of the $10 M_{\odot}$ black hole. Tidal distortion of the stellar photosphere is thought to be responsible for the (elipsoidal) modulation of the photometric lightcurves on the orbital period (Brocksopp et al. 2000). Distortion of the wind due to the gravitational potential of the black hole is also thought to increase the mass loss rate through the L1 point; the higher wind density (and hence emission measure) leading to the emission components visible in the $\mathrm{H} \alpha$ and He II lines. The lack of medium term photometric variability found for LS 5039 (Sect. 3.2) is thus consistent with a larger orbital radius proposed for the compact companion in this system by Paredes et al. (2000), which would naturally imply substantially reduced, or no, tidal interaction between both components of the binary (numerical simulations by Blondin et al. 1991, demonstrate that the distortion of the stellar wind and surface drop off rapidly with increasing orbital separation).
A large orbital separation also naturally explains the lower X-ray flux in LS 5039 compared to Cyg X-1 ( $\sim 2$ orders of magnitude; R99). Geometrical dilution will reduce the wind density at the proposed orbital separation of LS 5039 by $\sim 2$ orders of magnitude compared to that at the orbital radius of $\mathrm{Cyg} \mathrm{X}-1$, with a possible further reduction depending on the base density of the wind. Although the limited temporal sampling of both the photometric and radio lightcurves precludes the search for periodicities of $\leq 2$ days we believe that such a short orbital period is unlikely. In addition to the argument for a large orbital period of Paredes et al. (2000) accretion onto a possible White Dwarf companion in a close ( $P_{\text {orb }} \leq 2$ days $)$ orbit can be excluded - from Eq. (16) of Waters (1988) we find that the expected X-ray luminosity would be an order of magnitude smaller than that observed. We also note that such a short orbital period for a possible neutron star companion can also be excluded since the $\mathrm{O} 6.5 \mathrm{~V}((\mathrm{f}))$ star would be larger than the Roche Lobe (Eggleton 1983) leading to Roche Lobe overflow mass loss and a much larger X-ray luminosity than observed.

The lack of periodic variability in the X-ray lightcurve also suggests a low $(e<0.1)$ orbital eccentricity for LS 5039 given that accretion from a stellar wind with a $1 / r^{2}$ density distribution results in an accretion rate ranging from $\dot{M}_{0}(1-e)^{-2}$ to $\dot{M}_{0}(1+e)^{-2}$ (assuming on Bondi-Hoyle accretion theory). Given the long circularisation timescale for such a wide orbit (longer than the main sequence lifetime of an O6.5 star), this suggests that the system was left with such a low eccentricity after the supernova which produced the compact object.

Therefore, the low level aperiodic variability observed in the radio and X-ray wavebands is most likely due to inhomogineities or clumping in the outer stellar wind leading to changes in the accretion rate onto the compact companion (O star winds are known to be highly structured close to the $\operatorname{star}\left(<10 R_{*}\right)$ although their geometry at large radii are at present uncertain). We note however, that if such a large orbital radius is adopted then the relative velocity of the wind past the compact object - effectively $V_{\infty}$ - should preclude the formation of an accretion disc around it according to theories of standard direct wind fed accretion. Since the presence of an accretion disc is thought to be a prerequisite for the production of a collimated jet in X-ray binaries this is clearly problematic for such a large adopted orbital radius.

Finally, although apparently a direct wind fed system, LS 5039 clearly does not occupy either of the conventional Be or supergiant subclasses of HMXB. Negueruela \& Reig (2001) have remarked that there seem to be several such systems which either show hybrid properties of both subclasses or simply do not fit into either group.

\section{Conclusions}

We have presented optical and near-IR spectroscopy that confirm that the mass donor in LS 5039 is an early $\mathrm{O} 6.5 \mathrm{~V}((\mathrm{f}))$ star. Long term observations show that there 
are no long term trends in the optical properties of the $\mathrm{O} 6.5 \mathrm{~V}((\mathrm{f}))$ star. Likewise, while there are day to day variations of a few hundredths of a magnitude in the optical lightcurve we find no coherent periodicities or long term trends that might be the result of orbital modulation; the most likely explanations for the low level optical variability are changes in the mass loss rate or non radial pulsations in the system primary. Variations of $\sim 0.4 \mathrm{mag}$ are apparent in the $H$ and $K$ band photometry between 1995-2000; at present there are no obvious mechanisms for such variability within the system. Variability is also present in both X-ray and radio wavebands. However there is no evidence for significant flaring or periodic modulation; we note that if orbital modulation of the radio lightcurve was present above $\sim 4$ per cent of the mean flux level at $2.25 \mathrm{GHz}$ (as is seen in $\mathrm{Cyg} \mathrm{X}-1$ ) it would have been detectable. Although we cannot confirm the orbital separation suggested by Paredes et al. the lack of orbital modulation in the radio, $\mathrm{X}$-ray and optical lightcurves is consistent with a rather large orbital separation and hence long orbital period. We therefore attribute the differences in the properties of the LS 5039 and the similar system Cyg X-1 to a combination of the weaker stellar wind and larger orbital separation of LS 5039.

Acknowledgements. This paper is partially based on observations collected at the European Southern Observatory, Chile. The United Kingdom Infrared Telescope is operated by the Joint Astronomy Centre on behalf of the U.K. Particle Physics and Astronomy Research Council. Skinakas Observatory is a collaborative project of the University of Crete, the Foundation for Research and Technology-Hellas and the Max-PlanckInstitut für Extraterrestrische Physik. P. Reig acknowledges support via the European Union Training and Mobility of Researchers Network Grant ERBFMRX/CT98/0195.

\section{References}

Balona, L. A. 1992, MNRAS, 254, 404

Blondin, J. M., Stevens, I. R., \& Kallman, T. R. 1991, ApJ, 371,684
Blum, R. D., Ramond, T. M., Conti, P. S., Figer, D. F., \& Sellgren, K. 1997, AJ, 113, 1855

Brocksopp, C., Fender, R. P., Larionov, V., et al. 1999, MNRAS, 309, 1063

Clark, J. S., \& Steele, I. A. 2000, A\&AS, 141, 65

Clark, J. S., et al. 2000, A\&A, 356, 50

Drilling, J. S. 1975, AJ, 80, 128

Eggleton, P. P. 1983, ApJ, 268, 368

Gies, D. R., \& Bolton, C. T. 1986a, ApJ, 304, 371

Gies, D. R., \& Bolton, C. T. 1986b, ApJ, 304, 389

Hanson, M. M., Conti, P. S., \& Rieke, M. J. 1996, ApJS, 107, 281

Hanson, M. M., Rieke, G. H., \& Luhman, K. L. 1998, AJ, 116, 1915

Herbig, G. H. 1975, ApJ, 196, 129

Kilkenny, D. 1993, SAAOC, 15, 53

Kilkenny, D., van Wyk, F., Marang, F., Spencer Jones, J. H., \& Whittet, D. C. B. 1993, SAAOC, 15, 65 (K93)

Koorneef, J. 1983, A\&A, 128, 84

Lahulla, J. F., \& Hilton, J. 1992, A\&AS, 94, 265

Lamers, H. J. G. L. M., \& Leitherer, C. 1993, ApJ, 412, 771

Leitherer, C., Chapman, J. M., \& Koribalski, B. 1995, ApJ, $450,289 \mathrm{~L}$

Mathys, G. 1988, A\&AS, 76, 427

Martí, J., Paredes, J. M., \& Ribo, M. 1998, A\&A, 338, L71 (Ma98)

Motch, C., Haberl, F., Dennerl, K., Pakull, M., \& JanotPacheco, E. 1997, A\&A, 323, 853 (M97)

Negueruela, I. N., \& Reig, P. 2001, A\&A, submitted

Paredes, J. M., Marti, J., Ribo, M., \& Massi, M. 2000, Science, 288,2340

Pooley, G. G., Fender, R. P., \& Brocksopp, C. 1999, MNRAS, 302, L1

Predehl, P., \& Schmitt, J. H. M. M. 1995, A\&A, 293, 889

Puls, J., et al. 1996, A\&A, 305171

Puxley, P. J., Beard, S. M., \& Ramsay, S. K. 1992, in Data Analysis Workshop-4th ESO/ST-ECF Garching, 117

Ribó, M., Reig, P., Marti, J., \& Paredes, J. M. 1999, A\&A, 347,518 (R99)

Spencer Jones, J. H., Whittet, J. C. B., \& He, L. 1993, SAAOC, 15, 73 (SJ93)

Walborn, N. R., \& Fitzpatrick, E. L. 1990, PASP, 102, 379

Waters, L. B. F. M., Taylor, A. R., van den Heuvel, E. P. J., Habets, G. M. M. J., \& Persi, P. 1988, A\&A, 198, 200 